\title{
CONTRIBUCIÓN DE LA BIOANTROPOLOGÍA A LA RECONSTRUCCIÓN DE LOS PROCESOS PRODUCTIVOS PREHISTÓRICOS. EXOSTOSIS EN EL CANAL AUDITIVO EN LA POBLACIÓN PREHISPÁNICA DE GRAN CANARIA
}

\author{
CONTRIBUTION OF BIOANTHROPOLOGY TO THE RECONSTRUCTION OF \\ PREHISTORIC PRODUCTIVE PROCESSES. THE EXTERNAL AUDITORY EXOSTOSES IN \\ THE PREHISPANIC POPULATION OF GRAN CANARIA
}

\author{
JAVIER VELASCO VÁZQUEZ (*) \\ ERNESTO MARTÍN RODRÍGUEZ $(* *)$ \\ EMILIO GONZÁLEZ REIMERS $(* * *)$ \\ MATILDE ARNAY DE LA ROSA $(* * * *)$ \\ ANTONIO BETANCOR RODRÍGUEZ $(* * * * *)$
}

\begin{abstract}
RESUMEN
En el presente trabajo se pretende abordar el papel de los estudios bioantropológicos en la reconstrucción de los procesos productivos de las sociedades del pasado. Esta finalidad es perseguida a partir del examen y valoración de la prevalencia de exostosis óseas en el canal auditivo en la población prehistórica de Gran Canaria. Las exostosis auditivas constituyen una lesión ósea, bien documentada en trabajos experimentales y clínicos, estrechamente relacionada con la exposición del canal auditivo al agua fría. La estimación de esta anormalidad ósea en el conjunto poblacional analizado permite la definición de importantes variaciones territoriales en las estrategias económicas emprendidas por estos grupos humanos.
\end{abstract}

(*) Dpto. de Prehistoria y Arqueología. Universidad de Valladolid. Plaza del Campus, s/n. 47011 Valladolid. Correo electrónico: tibicena@inves.es

(**) Dpto. de Ciencias Históricas de la Universidad de Las Palmas de Gran Canaria. Pérez del Toro, 1. 35003 Las Palmas de Gran Canaria. Gran Canaria. Correo electrónico: emartin@sinf.ulpgc.es

(**) Dpto. de Medicina Interna. Hospital Universitario de Canarias. Universidad de La Laguna. La Laguna. Tenerife. Correo electrónico: egonrey@ull.es

$(* * * *)$ Dpto. de Prehistoria, Antropología e Historia Antigua. Universidad de La Laguna. Facultad de Geografía e Historia. Campus de Guajara. La Laguna. Tenerife.

(*****) Museo Canario. Doctor Chil. 35001 Las Palmas de Gran Canaria. Gran Canaria. Correo electrónico: abetancor@ elmuseocanario.com

El artículo fue remitido en su versión final el 15-I-2001.

\begin{abstract}
The aim of this paper is an approach to the role of bioanthropological studies in the reconstruction of the productive processes of past societies. This objective is obtained starting from the survey and valuation of the prevalence of bone exostoses in the auditory canal among the prehistoric inhabitants of Gran Canaria. The auditory exostose is a bone wound well documented through clinical and experimental studies, closely related to the exposure of the auditory canal to cold water. The estimation of this bone anomaly among the analysed population, leads to the definition of outstanding territorial variations in the economic strategies of these human groups.
\end{abstract}

Palabras clave: Bioarqueología. Prehistoria. Gran Canaria. Exostosis auriculares. Economía.

Key words: Bioarchaeology. Prehistory. Gran Canaria. Auditory exostoses. Economy.

\section{INTRODUCCIÓN}

Los estudios bioantropológicos en la actualidad parten de una concepción dinámica de los grupos humanos desde el punto de vista biológico y cultural, entendiendo a éstos como seres sensibles a los cambios que se producen en el entorno (natural y cultural) en el que desarrollan sus actividades. De esta manera se integra a las poblaciones del pasado 
en el marco de un contexto general que las define y de cuya dinámica de funcionamiento no permanecen ajenas. Estas valoraciones conceptuales han supuesto que, hoy por hoy, el estudio de los grupos humanos arqueológicos no se limite al análisis morfológico de sus restos o a la mera descripción y diagnóstico de patologías, sino que atienda también -y quizá de modo preferente- a las condiciones y calidad de vida de estas colectividades y al reflejo de éstas en las evidencias esqueléticas.

En otros términos, y al igual que sucede con la totalidad de los materiales arqueológicos, es necesario explicar qué posibles vínculos se establecen entre los restos arqueológicos y las actividades y relaciones sociales que las originaron o las modificaron (Bate, 1989). En los últimos años, uno de los retos más importantes a los que se enfrenta la investigación de las sociedades prehistóricas de Gran Canaria es la reconstrucción de lo que, en términos genéricos, se ha denominado "economía". Un campo de análisis que está siendo afrontado desde diversas disciplinas de estudio y, posiblemente lo que es más importante, desde unos planteamientos conceptuales diferentes, al menos, a los propuestos hasta el momento.

Producto de esta línea de investigación es la reciente publicación de diversos trabajos que, desde distintas perspectivas, han tratado de incidir en los aspectos que competen a esta materia de análisis. Es por este motivo que también se plantee como necesaria la valoración del papel que pueden desempeñar los estudios bioantropológicos en este sentido, un hecho especialmente relevante en este contexto insular, donde los exámenes centrados en los restos humanos de la población aborigen del Archipiélago cuentan ya con una tradición centenaria.

\section{EL MODELO PRODUCTIVO EN LA PREHISTORIA DE GRAN CANARIA Y LOS ANÁLISIS BIOANTROPOLÓGICOS}

Los procesos de producción (1) observables en esta sociedad prehistórica no pueden seguir considerándose tan sólo a partir de la estimación de uno o varios procesos de trabajo (agricultura, ganadería, recolección, etc.), sino mediante la valoración de las

(1) "El proceso productivo es el sistema orgánico de los diversos procesos de trabajo concretos a través de los cuales una sociedad genera las diversas clases de bienes que requiere para la satisfacción de las necesidades que permiten su mantenimiento y su reproducción y que está capacitada para producir" (Bate, 1988: 58).

T. P., 58, n. $^{\circ} 1,2001$ relaciones que mantienen los integrantes de estas comunidades entre sí, de los vínculos establecidos entre productores y no productores, así como de los elementos que configuran el control de los medios de producción y de los productos obtenidos en el desarrollo de tales actividades (rendimiento social del trabajo). Es por ello que necesariamente se ha de incluir en este análisis la unidad de procesos económicos básicos de cualquier sociedad, o lo que es lo mismo: producción, distribución, cambio y consumo, teniendo especialmente presente en este examen la organización social de la producción, esto es, la naturaleza del conjunto de relaciones sociales que conforman y definen las unidades básicas de producción de cada colectivo (Bate, 1998).

Es lógico entender por ello que las relaciones sociales de producción, o en otros términos la ordenación y normalización del proceso productivo en estas comunidades prehistóricas grancanarias, se conformarían esencialmente sobre la base de las reláciones de propiedad de los diferentes agentes que intervienen en el proceso de producción (Velasco et alii, 1999). Un hecho especialmente evidente si aceptamos que las formas de propiedad, como norma, se combinan con las formas específicas de organización del proceso de trabajo y de distribución de los productos así obtenidos. La valoración de las relaciones de propiedad en el caso de la Prehistoria de Gran Canaria presenta algunos elementos de necesaria discusión si atendemos a la información legada por las fuentes etnohistóricas referidas a estos grupos humanos. A este respecto Gómez Scudero señalará que "las tierras eran concejiles, que eran suias mientras duraba el fruto, cada año se repartían" (Morales, 1993: 436), asimismo López Ulloa añade nuevas consideraciones: "hera gente muy trabajadora, todos los bienes eran comunes en quanto a la distribución y alimento natural. Al señor reconocían la superioridad y obediencia y siempre se le daua lo mejor" (Morales, 1993: 315).

Los investigadores que han hecho referencia al control sobre los medios de producción en la sociedad prehistórica de Gran Canaria coinciden en señalar que sería el sector dirigente de esta sociedad (denominados "nobles") quienes controlarían plenamente su administración, a modo de "representantes" de la comunidad (González y Tejera, 1990; Jiménez, 1990). Mientras el grupo "dependiente", desprovisto de esta capacidad, no es más que el poseedor de unos derechos de uso sobre las propiedades objeto de redistribución. Con relación a ello, 
se ha señalado que de este modo "se garantiza a cada uno de sus miembros el acceso a la tierra y, por tanto, la supervivencia, proporcionando además a las generaciones posteriores la misma garantía" (González y Tejera, 1990: 116).

Sin embargo, esta realidad socioeconómica parece mostrar un mayor grado de complejidad, permitiendo una interpretación parcialmente diferenciada a la recogida en párrafos precedentes. En ese sentido, cabe destacar como, en primer lugar, el control y la administración del grupo dirigente de los derechos sobre el territorio reglamenta socialmente la desigualdad en el acceso a los medios de producción básicos para el desarrollo de los procesos productivos. Ello, y en segundo lugar, no hace más que reforzar la diferenciación en la capacidad de acumular y reproducir el producto social del trabajo. Estas limitaciones, reconocidas y sancionadas por toda la comunidad (2), constituyen uno de los elementos claves que justifican, y a la vez aseguran, la existencia de unas relaciones sociales de dependencia.

Los sectores dominantes, por tanto, ejercen una evidente preeminencia en la utilización y acceso al objeto de trabajo y a los instrumentos de producción que capacitan su transformación, lo que les facultará simultáneamente para el dominio y el control efectivo de la totalidad del proceso productivo. En este sentido resulta posible plantear entonces que el acceso de los sectores dependientes a los recursos que aseguren su subsistencia no está garantizado por su simple pertenencia a la comunidad, sino por el mantenimiento de unas relaciones sociales de dependencia con los grupos que detentan el control directo de los medios de producción. Es factible afirmar, por tanto, y como ya han sugerido otros autores, que estos vínculos de dependencia se desarrollan y consolidan a través de la propia estructura de producción, especialmente a partir de la apropiación social del producto (Hernández y Galván, 1997; Velasco y Martín, 1998).

Difícilmente, y en vista a los planteamientos expuestos, puede seguir manteniéndose la valoración de los procesos productivos de la sociedad prehistórica de Gran Canaria teniendo como único referente la estimación de los procedimientos téc-

(2) En este sentido cabe señalar como el grupo no detentador de los medios de producción "acepta", por así decir, la existencia de estas relaciones de desigualdad, siempre y cuando éstas se conciban como un servicio prestado por parte del sector dominante de estas sociedades, "cuyo poder parece que desde ese momento es tan legítimo para los dominados como su propio deber de servir ellos a quienes les sirven" (Godelier, 1989: 188) nicos seguidos para la obtención de los productos que garanticen la subsistencia de estos grupos. Con relación a ello, resulta también evidente que los procesos productivos de las sociedades a las que aquí aludimos no pueden seguir definiéndose bajo el concepto de "economías de autosubsistencia o autosuficiencia". De ser así quedaría enmascarado el hecho de que la actividad productiva no se limita a la obtención de bienes de subsistencia, sino que también es la encargada de lograr un plusproducto destinado al desarrollo y consolidación de las propias estructuras sociales, ocultándose, de igual modo, las numerosas formas de cambio e intercambio que son consustanciales a este funcionamiento (Godelier, 1977).

Una prueba de tal circunstancia lo constituye la existencia en Gran Canaria, según manifiestan las noticias etnohistóricas (3), de una parte de la población que no participa directamente en la producción de los bienes alimenticios básicos. Su existencia requeriría, por tanto, que los "productores directos" transfirieran parte del rendimiento de su trabajo para el mantenimiento de aquellos, lo que no puede entenderse sin la existencia, al menos en este marco, de un sistema jerarquizado de toma de decisiones en la ordenación global del modelo productivo. Tales datos, por ello, hacen alusión directa a la existencia de una división social del trabajo. A tal efecto, la organización de la producción pasa por el establecimiento y ordenación de determinadas actividades cuyo fin último parece ser la consolidación y perpetuación del modelo organizativo, así como de las relaciones sociales que lo hacen posible.

No obstante, las referencias proporcionadas por los cronistas de la Conquista e historiadores posteriores son sumamente escuetas en el sentido propuesto, aportando referencias como las que siguen: "los canarios tenían entre ellos oficiales de hacer casas debajo y encima de la tierra, carpinteros, sogueros" (Torriani, 1978: 112); "tenían casas y oficiales que las hacían de piedra seca" (Abreu, 1977: 159). En algunos casos, esta división del trabajo parece que está estrechamente vinculada a las diferencias de género: "tenían mujeres dedicadas para sastres, como para hacer loza de que usaban que eran tallas como tinajuelas para agua" (Morales, 1993: 371). De igual modo, ciertos "oficios" llevan aparejados un tabú de contacto, como es el caso de los carniceros o los verdugos: "para cuyo

(3) Algunas pruebas arqueológicas parecen venir a confirmar tal extremo de forma inequívoca como se ha propuesto, por ejemplo, para el caso de Tenerife (Hernández y Galván, 1997). 
efecto tenían berdugos diputados con salario, que acudian a todo lo que se les mandaba" (Morales, 1993: 313).

Las pruebas arqueológicas que puedan corroborar la existencia de esta particular ordenación del proceso productivo son escasas, por el momento, ya que su estimación va en consonancia con la progresiva reactivación de intervenciones arqueológicas en Gran Canaria. A pesar de ello, los análisis sobre restos humanos han empezado en fechas recientes a aportar nuevos elementos de juicio sobre los que estimar los planteamientos hechos hasta el momento.

Este tipo de estudios tiene la ventaja de incidir plenamente sobre los protagonistas directos de los comportamientos a los que se alude, lo que conlleva la evaluación de estos procesos desde una óptica biológica y cultural. En el mismo sentido, facilitará en los casos en los que sea posible la caracterización de un conjunto poblacional amplio, permitiendo observar así tanto la generalización de unas pautas de conducta, como su propia diversificación en el marco definido por los factores espacio, tiempo y cultura. En este orden de cosas, hay que manifestar que los datos derivados de este tipo de estudios no pueden pasar a entenderse por sí solos, sino que habrán de ser estimados en estrecha relación con el contexto cultural, cronológico y espacial del que provienen los materiales objeto de examen. El conocimiento de este entorno proporcionará buena parte de los criterios básicos que permitan considerar el grado de representatividad y la significación de los resultados obtenidos mediante la investigación bioantropológica.

\section{MARCADORES ÓSEOS DE ACTIVIDAD FÍSICA: ¿EVIDENCIAS BIOANTROPOLÓ- GICAS DE LA ORDENACIÓN DEL PROCE- SO PRODUCTIVO?}

Dentro de las líneas de investigación preferentes en la ciencia bioantropológica actual pueden enmarcarse los exámenes de repertorios óseos que tienen como fin último hacer un balance global de aquellas evidencias de actividades físicas observables en el esqueleto. Siguiendo las valoraciones de K. Kennedy (1989), tales marcadores son una expresión evidente de la plasticidad ósea ante presiones extracorporales, así como bajo la influencia de otras fuerzas "internas" que no son atribuibles a alteraciones patológicas, metabólicas, bioquímicas, hormona- les, a los intercambios enzimáticos o anomalías de orden vascular o neuronal.

Durante los últimos años las publicaciones sobre esta materia han proliferado de forma significativa, acompañadas, además, de un amplio debate en torno a la potencialidad y limitaciones que presenta esta línea de estudio. Parece existir un cierto asenso entre los investigadores a la hora de señalar las dificultades que supone la correlación directa de una alteración ósea con una actividad física u ocupacional particular, si bien es igualmente cierto que permite vincular unos caracteres observables en el esqueleto con la reacción del organismo ante determinados estímulos ambientales (Stirland, 1992; Dutour, 1992). Sin duda, la valoración del contexto arqueológico-cultural del que proceden los materiales sujetos a observación aportará los criterios más idóneos para el establecimiento de las distintas hipótesis interpretativas.

Un ejemplo evidente en este sentido puede venir aportado por el estudio de las exostosis del canal auditivo en la población prehispánica de Gran Canaria.

\subsection{Exostosis del canal auditivo: ¿alteraciones "profesionales"?}

Estas exostosis corresponden a hiperplasias óseas de crecimiento que pueden formarse en el tracto medio o en la entrada del conducto auditivo externo, conformando una anomalía característica ya observada por diversos investigadores desde fines de la pasada centuria. Estas excrecencias óseas, más o menos compactas y con una estructura fundamentalmente laminar, han recibido la denominación de torus auditivus o exostosis (Gervais, 1989; Manzi et alii, 1991; Deleyianis et alii, 1996), si bien este último término es el más generalizado en la literatura especializada. Morfológicamente muestran una apariencia oval o esferoide, constatándose en menor proporción otras tipologías. A pesar de que este tipo de exostosis ha sido eventualmente incluida en las listas de los caracteres no métricos del cráneo (Brothwell, 1987), en la actualidad se cuestiona su naturaleza epigenética toda vez que se ha comprobado la naturaleza no hereditaria de este carácter y la estrecha relación entre la prevalencia de esta anomalía y los factores medioambientales que la provocan (Kennedy, 1986; Manzi et alii, 1991; Karegeannes, 1995; Hutchinson et alii, 1997; Ito e Ikeda, 1998). 
El número, la lateralidad, el volumen y la morfología de estas exostosis del canal auditivo pueden llegar a ser ciertamente variables, constatándose, incluso, ciertas divergencias en un mismo individuo. En la mayor parte de los sujetos suelen tener un carácter único, si bien resulta posible la detección de hasta cuatro focos de reacción ósea. Con relación a ello, el volumen que adquieren variará en un amplio espectro, llegando a darse el caso -frecuentemente en los sujetos de mayor edad-de una obstrucción prácticamente completa del conducto auditivo (Gerszten et alii, 1998; Whitaker et alii, 1998). La bilateralidad de esta anomalía concierne, cono norma, a más del $50 \%$ de los individuos afectados.

La posibilidad de vincular esta anomalía ósea con determinadas actividades desarrolladas por las poblaciones del pasado viene propiciada por las causas que provocan su aparición. Partiendo de los resultados obtenidos en diversos trabajos experimentales, así como mediante observaciones clínicas, la etiología hidrotérmica de estas exostosis se encuentra plenamente reconocida en los estudios de patología humana, en detrimento de otras reacciones hiperostósicas más difusas, esporádicas y que suelen estar acompañadas de procesos infecciosos crónicos (Kennedy, 1986; Manzi et alii, 1991; Dastugue y Gervais, 1992; Standen et alii, 1997; Deleyianis et alii, 1996).

Según recogen G. Kennedy (1986) existe una estrecha relación entre el grado de desarrollo de estas exostosis y la frecuencia de la exposición del canal auditivo al agua fría (4). De este modo, se ha propuesto que la vasodilatación resultante de dicha situación provocaría una excitación local del perióstilo y, por ello, una estimulación anormal de la actividad osteoblástica (creación de tejido óseo) (Kennedy, 1986; Gervais, 1989; Karegeannes, 1995; Standen et alii, 1997; Deleyianis et alii, 1996; Ito e Ikeda, 1998). La continuidad en el desarrollo de los hábitos que comportan este hecho, conlleva la repetición de la dinámica descrita y, por ello, la sucesión de reacciones exostósicas locales (5). En relación con este último punto, se ha descrito como los sujetos infantiles y adolescentes suelen estar exentos de esta anormalidad, mientras que a medida

(4) Normalmente por debajo de los $19^{\circ} \mathrm{C}$ (especialmente entre los $15^{\circ} \mathrm{C}$ y $19^{\circ} \mathrm{C}$ ) (Kennedy, 1986).

(5) Si bien la relación etiológica ente la aparición de exostosis y el contacto del canal auditivo con el agua fría constituye un hecho demostrado, ha de mantenerse cierta prudencia en la valoración de esta entidad a consecuencia de la diversidad de agentes causales que, en más de una ocasión, pueden estar interactuando (Tran et alii, 1996; Hutchinson et alii, 1997). que avanzan en la edad adulta, y continua la exposición del canal auditivo al agua fría, los procesos exostósicos ven incrementado su volumen, y quizá su número, de forma progresiva.

J. Dastugue y V. Gervais (1992) incluyen estas neoproducciones óseas dentro de lo que califican como "enfermedades profesionales". La razón que les lleva a mantener tal postura es que, fuera de toda duda, para la aparición y desarrollo de tales lesiones el contacto y/o la inmersión en agua fría debe ser frecuente, regular y suficientemente prolongada. De este modo, ha sido puesta en relación con poblaciones en las que el contacto directo con el medio acuático constituiría una parte fundamental de sus actividades cotidianas, especialmente en lo que se refiere a la búsqueda y obtención de recursos alimenticios procedentes de estos entornos (mar, ríos, lagos, etc.). En este sentido, estudios desarrollados en poblaciones actuales en las que se da un importante contacto con el medio acuático (submarinistas, practicantes desurf, etc.) han podido constatar un aumento significativo de la presencia de este tipo de anormalidades óseas a medida que se incrementan los años dedicados a tal actividad con una frecuencia constante (Umeda et alii, 1989; Karegeannes, 1995; Deleyianis et alii, 1996 (6); Ito e Ikeda, 1998). Algunos estudios con materiales arqueológicos han permitido constatar esta circunstancia (Kennedy, 1986; Sakalinskas y Jankauskas, 1993; Hutchinson et alii, 1997; Standen et alii, 1997), manifestando, en el mismo sentido la estrecha asociación que existe entre la presencia de exostosis auriculares y el desarrollo de actividades de explotación económica de los medios acuáticos.

Para el caso concreto de Canarias, la presencia de estas exostosis auriculares fue observada y descrita por O. Dutour y J. Onrubia (1991) en los restos esqueléticos exhumados de la Necrópolis de El Agujero (Gáldar). En tal caso se señalaba la estrecha relación de los habitantes de esta zona de Gran Canaria con el medio oceánico, manifestándose igualmente la doble vertiente lúdica y económica que tendría esta actividad. Resultaba, asimismo, muy significativa la prevalencia de esta anormali-

(6) De este modo, en un estudio desarrollado entre "surferos" de Oregón, se pone de manifiesto como el $63 \%$ de los individuos que han practicado este deporte entre 6 y 15 años presentan exostosis del canal auditivo, incrementándose este porcentaje hasta un 93\% cuando esta actividad se ha prolongado más de una quincena de años. Concluye este trabajo señalándose que los sujetos que han practicado surf cinco años o menos no suelen presentar esta anormalidad ósea en el canal auditivo, a no ser que tal actividad se haya desarrollado en más de cincuenta sesiones anuales. 


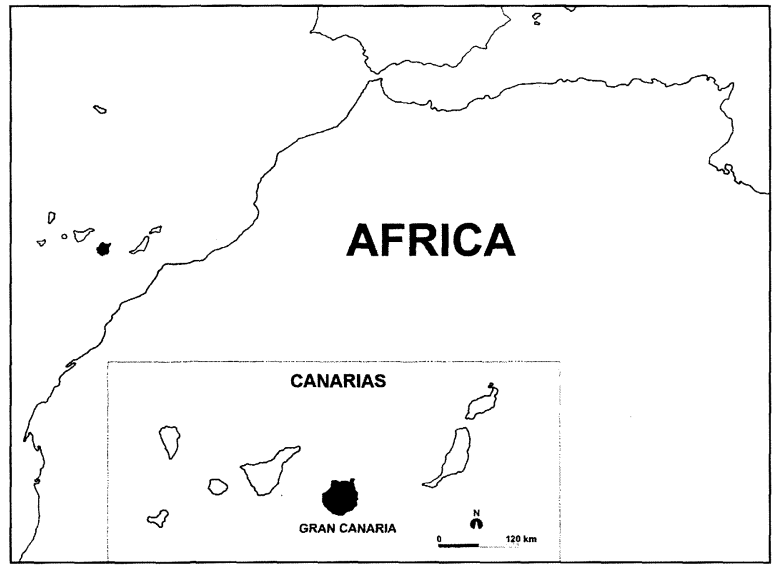

Fig. 1. Localización geográfica del Archipiélago Canario y de la isla de Gran Canaria.

dad en el subconjunto poblacional estudiado, que superaba netamente los valores obtenidos en otras áreas del planeta, lo cual vendría a ratificar la relevancia de esta relación grupo-entorno marino.

Trabajos más recientes, afrontados desde otras perspectivas de análisis, han puesto en evidencia el estrecho vínculo que uniría a los canarios con el mar. La recolección marina y especialmente, a juzgar por los datos disponibles, la pesca constituirían estrategias económicas de suma importancia para la subsistencia de estas poblaciones (Rodríguez, 1996; Velasco et alii, 1997). Cabe señalar que no se trata de estimar la explotación del medio marino como una mera actividad encaminada a obtener unos recursos alimenticios más o menos apetecibles. En términos reales esta estrategia contribuiría a la consolidación del modelo agrícola y ganadero de Gran Canaria, minimizando los efectos negativos de cualquier alteración de los ciclos productivos y reforzando la dependencia del grupo con respecto a las prácticas productivas.

A fin de profundizar en los planteamientos hechos en páginas precedentes, se afronta el presente trabajo.

\section{MATERIAL Y MÉTODO}

Se estudiaron un total de 358 cráneos, todos ellos correspondientes a sujetos adultos, procedentes de 27 contextos sepulcrales de Gran Canaria (7) (Fig. 1). El criterio para su inclusión fue que presentaran un nivel de conservación tal que fuera posible

(7) Todos ellos custodiados en los fondos de El Museo Canario de Las Palmas de Gran Canaria. la determinación del sexo y la edad aproximada a la que aconteció su muerte. Dados los objetivos de la investigación, se tuvo en cuenta, además, que en cada uno de ellos resultara factible la observación de los dos canales auditivos.

El sexo de todos los sujetos fue establecido siguiendo los criterios morfológicos recogidos por W. Krogman y M.Yasar Iscan (1989), siendo determinados 200 hombres, 144 mujeres, así como 14 que fueron clasificados como alofisos.

La asignación de los grupos de edad se realizó mediante la observación del grado de desgaste de las piezas dentarias, siguiendo la propuesta de D.R. Brothwell (8) (1987, 1989; Perizonius, 1983) y, ya que la muestra se encontraba formada exclusivamente por individuos adultos, se utilizaron intervalos amplios de edad: 17-24, 25-34, 35-45 y <45 (9). Esta información fue contrastada con la observación del grado de sinostosis de las suturas craneales, obteniéndose un óptimo grado de correlación entre ambos marcadores en la mayor parte de los casos.

Los canales auditivos fueron inspeccionados visualmente con luz directa y con la ayuda de una lente de 20 aumentos a fin de verificar la presencia o ausencia de exostosis auriculares. Se llevó a cabo una valoración macroscópica de los procesos exostósicos, ordenándolos tipológicamente siguiendo los parámetros propuestos por V. Gervais (1989) (10). Para la consideración del volumen que ocuparían se establecieron tres categorías diferenciadas: $<1 / 3,1 / 3-2 / 3$ y $>2 / 3$ del total del canal auditivo (Standen et alii, 1997). En la descripción se tuvo igualmente en cuenta la lateralidad de las reacciones exostósicas observables, así como la posición ocupada por éstas en la pared del canal auditivo (11).

Todos los datos, recogidos en fichas normalizadas, fueron incorporados para su análisis en el pa-

(8) En el mismo sentido, se observó la morfología del desgaste a fin de no estimar en la valoración de este parámetro los grados de atricción anómalos provocados por procesos patológicos como la precoz pérdida antermorten de molares, o los desgastes originados por el empleo de estas piezas en procesos de trabajo como se ha constatado en algunos individuos (Velasco $e t$ alii, 2000).

(9) La elección de intervalos amplios de edad minimizaba los posibles errores que pudieran derivarse de tal observación a consecuencia de las peculiaridades que presenta el patrón de desgaste dentario de las poblaciones prehistóricas de las Islas.

(10) Esta autora propone el establecimiento de cuatro tipos fundamentales: laminar, nodular, en espina y en mamelón.

(11) Para ello se tomaron como referencia las siguientes posiciones en la pared del canal auditivo: anterior, posterior, anterosuperior, anteroinferior, posterosuperior y posteroinferior. 


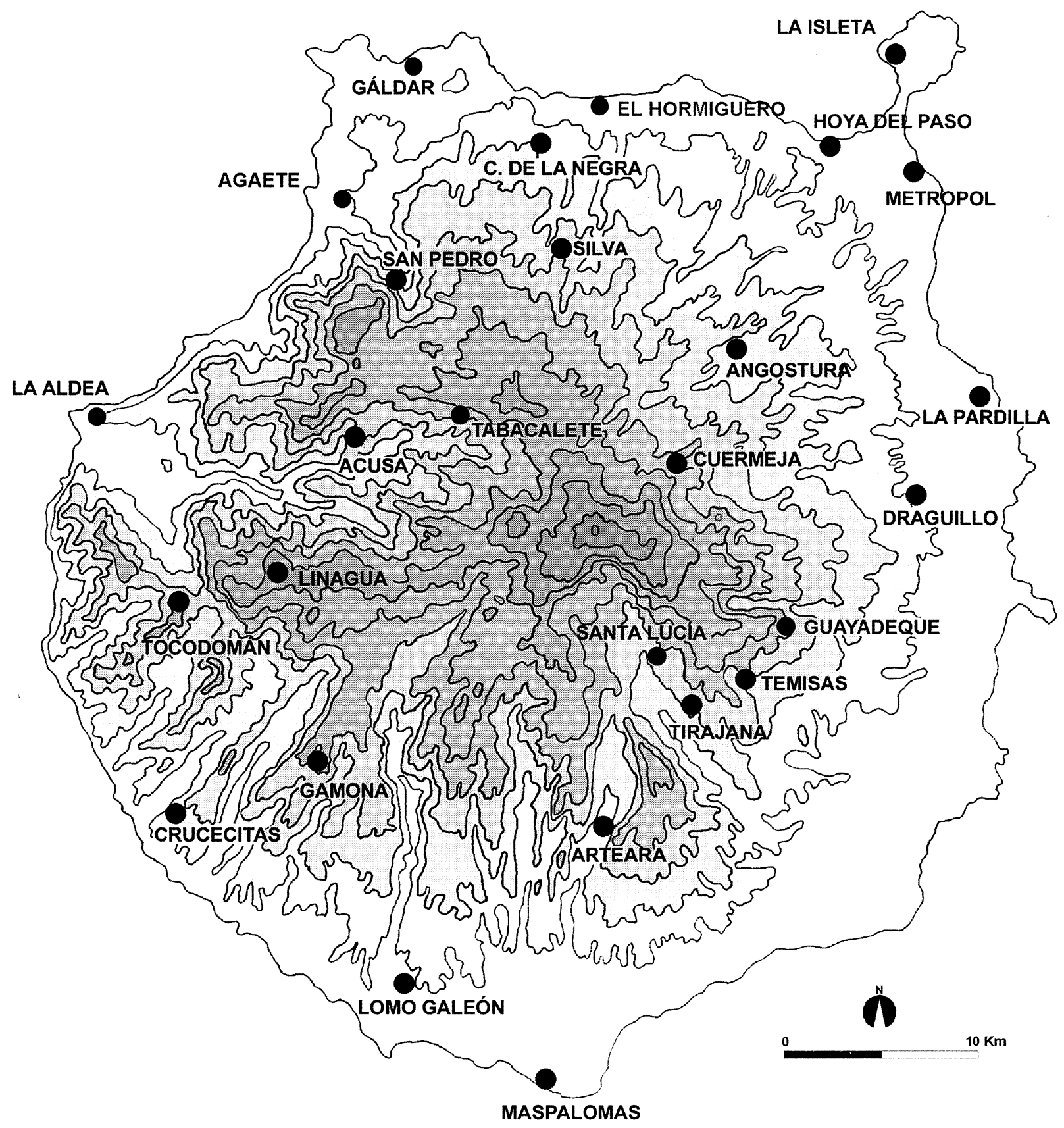

Fig. 2. Distribución espacial de los yacimientos estudiados en Gran Canaria.

quete estadístico SPSS 8.0. Las pruebas de significación estadística, para comparación de grupos (distribución de frecuencias), se realizaron mediante la aplicación de la prueba de chi-cuadrado $\left(\mathrm{X}^{2}\right)$. Igualmente se empleó el coeficiente de correlación r de Pearson para medir la magnitud de la relación entre dos variables. En todos los casos el nivel de significación estadística se fijó en $\mathrm{p} \leq 0,05$.

\subsection{Los contextos arqueológicos}

Los contextos sepulcrales estudiados se encuentran repartidos por la geografía de Gran Canaria, hallándose tanto en las proximidades de la línea de costa, como en el interior de la Isla (Fig. 2). Lo yacimientos valorados y el número de individuos procedente de cada uno de ellos se exponen en la tabla 1. 


\begin{tabular}{|c|c|c|c|c|}
\hline YACIMIENTO & Indv. afectados/Total indv. & Hombres & Mujeres & Indeterminados \\
\hline \multicolumn{5}{|c|}{ ÁMBITO COSTERO } \\
\hline Agaete & $2 / 6(33.3 \%)$ & $2 / 5(40 \%)$ & $0 / 1$ & - \\
\hline Aldea & $1 / 11(9.1 \%)$ & $0 / 6$ & $1 / 5(20 \%)$ & - \\
\hline Crucecitas & $3 / 14(21.4 \%)$ & $2 / 10(20 \%)$ & $1 / 4(25 \%)$ & - \\
\hline Cuesta de la Negra & $0 / 2$ & $0 / 1$ & - & $0 / 1$ \\
\hline Draguillo & $2 / 11(18.18 \%)$ & $2 / 5(40 \%)$ & $0 / 6$ & - \\
\hline Gáldar & $25 / 42(59.52 \%)$ & $16 / 26(61.53 \%)$ & $9 / 16(56.25 \%)$ & - \\
\hline Hormiguero & $0 / 13$ & $0 / 11$ & $0 / 2$ & - \\
\hline Hoya del Paso & $1 / 4(25 \%)$ & $1 / 3(33,33 \%)$ & $0 / 1$ & - \\
\hline Isleta & $0 / 14$ & $0 / 7$ & $0 / 7$ & - \\
\hline Lomo Galeón & $3 / 4(75 \%)$ & $3 / 3(100 \%)$ & $0 / 1$ & - \\
\hline Maspalomas & $0 / 1$ & - & $0 / 1$ & - \\
\hline Metropol & $7 / 8(87.5 \%)$ & $3 / 3(100 \%)$ & $4 / 5(80 \%)$ & - \\
\hline Pardilla & $1 / 1(100 \%)$ & $1 / 1(100 \%)$ & - & - \\
\hline TOTAL COSTA & $45 / 131(34.35 \%)$ & $30 / 81(37.03 \%)$ & $15 / 49(30.61 \%)$ & $0 / 1$ \\
\hline \multicolumn{5}{|c|}{ "INTERIOR" } \\
\hline Acusa & $1 / 20(5 \%)$ & $1 / 10(10 \%)$ & $0 / 10$ & - \\
\hline Angostura & $0 / 9$ & $0 / 6$ & $0 / 3$ & - \\
\hline Arteara & $0 / 6$ & $0 / 4$ & $0 / 2$ & - \\
\hline Cuermeja & $1 / 1(100 \%)$ & - & $1 / 1(100 \%)$ & - \\
\hline Gamona & $0 / 1$ & - & $0 / 1$ & - \\
\hline Guayadeque & $0 / 98$ & $0 / 45$ & $0 / 44$ & $0 / 9$ \\
\hline Linagua & $0 / 7$ & $0 / 5$ & $0 / 1$ & $0 / 1$ \\
\hline San Pedro & $0 / 2$ & $0 / 2$ & - & - \\
\hline Santa Lucía & $0 / 8$ & $0 / 7$ & $0 / 1$ & - \\
\hline Silva & $0 / 2$ & $0 / 2$ & - & - \\
\hline Tabacalete & $0 / 33$ & $0 / 16$ & $0 / 16$ & $0 / 1$ \\
\hline Temisas & $0 / 8$ & $0 / 3$ & $0 / 4$ & $0 / 1$ \\
\hline Tirajana & $0 / 31$ & $0 / 18$ & $0 / 12$ & $0 / 1$ \\
\hline Tocodomán & $1 / 1(100 \%)$ & $1 / 1(100 \%)$ & - & - \\
\hline TOTAL INTERIOR & $3 / 227(1.32 \%)$ & $2 / 119(1.68 \%)$ & $1 / 95(1.05 \%)$ & $0 / 13$ \\
\hline TOTAL GLOBAL & $48 / 358(13.4 \%)$ & $32 / 200(16 \%)$ & $16 / 144(11.1 \%)$ & $0 / 14$ \\
\hline
\end{tabular}

Tab. 1. Material sujeto a examen y prevalencia de exostosis en la población prehispánica de Gran Canaria por yacimiento (Indv. Individuos).

A fin de explicar la incidencia de exostosis auriculares en esta muestra de población, se procedió a su ordenación por comarcas naturales (12), dividiéndolos, en función de su proximidad al entorno litoral, entre conjuntos costeros e "interiores". Si bien esta clasificación puede ser cuestionable en un territorio con una extensión tan limitada (1500 $\mathrm{km}^{2}$ ), la información proporcionada por los estudios de elementos traza manifestaba la existencia de

(12) Con ello se pretendía, igualmente, minimizar las diferencias en el número de individuos observados en cada uno de los espacios sepulcrales y los problemas que ello entrañaría en el análisis estadístico. diferencias significativas en el acceso a los recursos marinos entre las poblaciones asentadas en una $\mathrm{u}$ otra zona de Gran Canaria (Velasco et alii, 1997).

Los criterios para proponer tal distinción responden tanto a la inmediatez de las áreas sepulcrales al litoral grancanario (13), como las condiciones de accesibilidad a éste desde los lugares en los que se ubican las zonas de asentamiento vinculadas a cada una de las necrópolis. Evidentemente, se trata de una división hasta cierto punto ficticia toda vez que la información arqueológica manifiesta que no debió ser la relación de proximidad el único parámetro que condicionaría la relación de los canarios con 
los recursos ofertados por el medio marino. Tan sólo constituye una propuesta por la que se pretende ofrecer una primera ordenación de los materiales estudiados a fin de estimar las condiciones etiológicas de la aparición y desarrollo de estas neoformaciones óseas, así como aportar nuevos puntos de vista a la ordenación territorial del modelo productivo de esta sociedad prehistórica.

De este modo, quedaron incluidos en el grupo costero los siguientes enclaves: Gáldar, Agaete, La Aldea, Metropol, La Isleta, Lomo Galeón, Cuesta de la Negra, Maspalomas, Crucecitas, Draguillo, Hoya del Paso, La Pardilla y El Hormiguero.

\section{RESULTADOS}

Pudo observarse la presencia de exostosis auriculares en 48 de los 358 individuos estudiados (Tab. 1), lo que viene a significar un $13.4 \%$ del total (Lám. I). El porcentaje relativamente elevado de exostosis auriculares en esta población permite clasificarla, según la propuesta de G. Kennedy (1986), dentro de los conjuntos poblacionales con una incidencia "media" de este marcador bioantropológico. Entre los hombres, la prevalencia de esta anormalidad ósea concierne a un 16\% (32/200), ligeramente superior al determinado para las mujeres: $11.11 \%(16 / 144)$, al igual que se ha descrito para otras poblaciones (Sakalinskas y Jankauskas, 1993; Standen et alii, 1997), si bien constituyen desemejanzas que no muestran significación estadística $\left(\mathrm{X}^{2}=3.98 ; \mathrm{p}=0.137\right.$; n.s. $)$

La bilateralidad de las exostosis auriculares afecta al 79.2\% del conjunto de población en la que pudo determinarse su presencia, siendo ligeramente más elevados los casos pertenecientes al género masculino (66.7\%) que los femeninos (31.6\%). En lo que se refiere a la localización (Tab. 2), cabe indicar que, como se ha descrito en otros casos (Gervais, 1989; Manzi et alii, 1991; Standen et alii, 1997), estas hiperplasias óseas suelen encontrarse ubicadas preferentemente en la superficie posteroinferior del canal auditivo externo (77\%), si bien también se situaban en una proporción considerable en la región anterior y anterosuperior del mismo ( $4.16 \%$ y $14.58 \%$ respectivamente).

La tipología de las exostosis auriculares observadas (Tab. 3) es predominantemente nodular, aun-

(13) La estrecha relación espacial entre espacios habitacionales y áreas de necrópolis permite tal distinción.

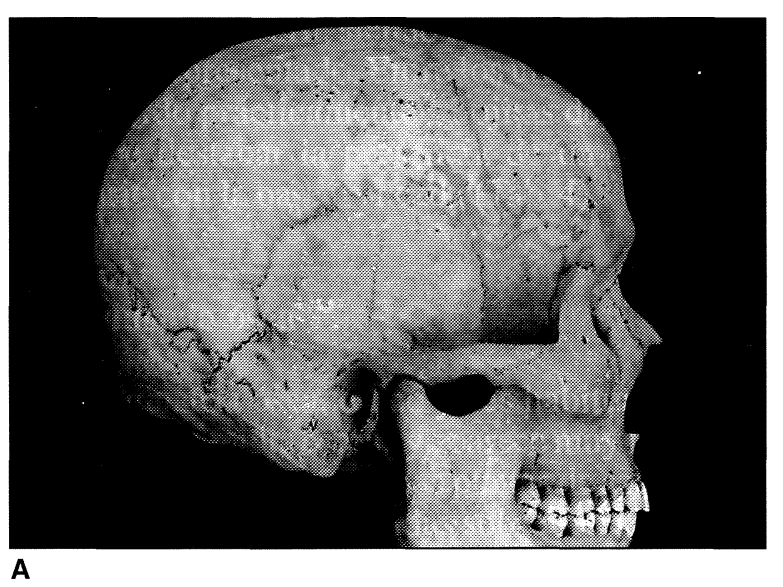

A

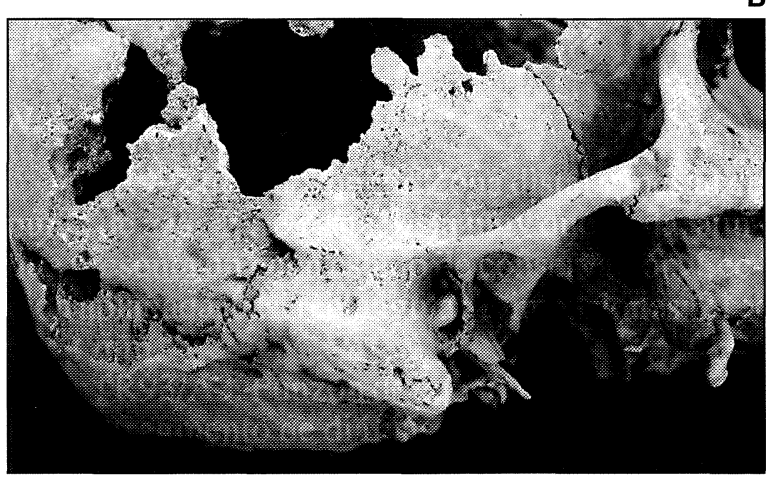

Lám. I. A Exostosis en el canal auditivo derecho de dos individuos masculinos procedentes de Gáldar (Gran Canaria). En el caso reproducido en $\mathrm{B}$, la neoproducción ósea ocupa más de dos tercios del canal auditivo.

que también están presente en un número elevado de casos las formas mamelonadas. En un porcentaje muy significativo de los sujetos sometidos a examen ambas tipologías están presentes de forma simultánea, variando su localización en el canal auditivo sin guardar un patrón normalizado. No existen, con relación a este parámetro, diferencias entre ambos sexos.

\begin{tabular}{|c|c|c|}
\hline Localización & $\mathbf{n}$ & $\mathbf{\%}$ \\
\hline Anterior & 2 & $4.16 \%$ \\
\hline Anteroposterior & 1 & $2.08 \%$ \\
\hline Anterosuperior & 7 & $14.58 \%$ \\
\hline Posterior & 0 & - \\
\hline Posteroinferior & 37 & $77 \%$ \\
\hline Posterosuperior & 1 & $2.08 \%$ \\
\hline
\end{tabular}

Tab. 2. Localización de las exostosis en la pared del canal auditivo de la población prehispánica estudiada de Gran Canaria. 


\begin{tabular}{|c|c|c|c|}
\hline Tipología & $\mathbf{n}$ & Hombres & Mujeres \\
\hline Nodular & $37(77.08 \%)$ & $23(71.87 \%)$ & $14(87.5 \%)$ \\
\hline Mamelonada & $9(18.75 \%)$ & $7(21.87 \%)$ & $2(12.5 \%)$ \\
\hline Laminar & $2(4.16 \%)$ & $2(6.25 \%)$ & - \\
\hline
\end{tabular}

Tab. 3. Tipología de exostosis en la población prehispánica estudiada de Gran Canaria.

\begin{tabular}{|c|c|c|c|c|}
\hline \multirow{2}{*}{ Edad } & \multicolumn{3}{|c|}{ Volumen exostosis } & \multirow{2}{*}{ Totales } \\
\cline { 2 - 4 } & $<\mathbf{1 / 3}$ & $\mathbf{1 / 3 - 2 / 3}$ & $\mathbf{2 / 3}$ & \\
\hline $17-25$ & $11(78.57 \%)$ & $1(7.14 \%)$ & $2(14.28 \%)$ & 14 \\
\hline $25-35$ & $5(41.66 \%)$ & $2(16.66 \%)$ & $5(41.66 \%)$ & 12 \\
\hline $35-45$ & $7(58.33 \%)$ & $3(35 \%)$ & $2(16.66 \%)$ & 12 \\
\hline$>45$ & $4(40 \%)$ & $1(10 \%)$ & $5(50 \%)$ & 10 \\
\hline Total & $27(56,25 \%)$ & $7(14.58 \%)$ & $14(29.16 \%)$ & 48 \\
\hline
\end{tabular}

Tab. 4. Volumen de los procesos exostósicos por grupos de edad en la población prehispánica estudiada de Gran Canaria.

La consideración del volumen de estas excrecencias óseas (Tab. 4) aporta datos de gran relevancia para estimar la significación de la presencia de estas anomalías en el repertorio esquelético valorado (Lám. II). Existe una relación evidente entre las dimensiones de las anomalías descritas y el momento de la muerte del individuo. Los valores logrados muestran una tendencia de incremento porcentual en el volumen ocupado por las exostosis auriculares en el canal auditivo a medida que se avanza en cada uno de los grupos de edad establecidos. De este modo, en aquellos sujetos mayores de 45 años las exostosis que ocupan más de las dos terceras partes del espacio disponible, en al menos uno de los canales auditivos, suponen un 50\% del total; mientras, este porcentaje se reduce sensiblemente en los individuos fallecidos entre 17-25 años (14.28\%), incrementándose en el grupo de 25-35 (41.66\%) (14). En el mismo sentido, en el $78.6 \%$ de

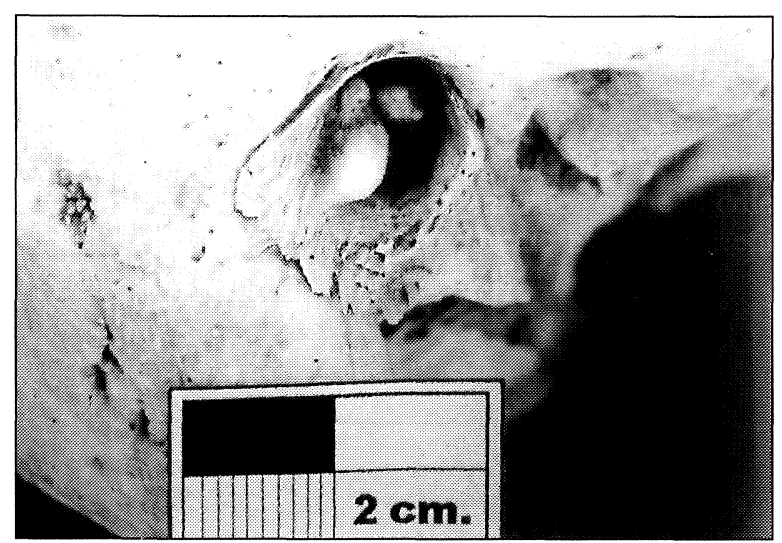

Lám. II. Detalle de exostosis en el canal auditivo del lateral derecho de un individuo masculino. los individuos con exostosis fallecidos entre los 17 y los 25 años, estas excrecencias ocuparían menos de un tercio del canal auditivo, observándose con ello una relación coherente entre edad de muerte y tamaño de las excrecencias óseas. En otros términos, se percibe una tendencia por la que las neopruducciones óseas en el canal auditivo tendrían un volumen más importante cuanto mayor sea la edad del sujeto afectado. Estos datos, previsiblemente, vienen a confirmar la continuidad en los hábitos que inducen la aparición y desarrollo de este marcador en la población sometida a estudio (15).

Los resultados obtenidos en cada uno de los conjuntos arqueológicos contribuyen significativamente a explicar la presencia de esta anomalía en los restos esqueléticos analizados, como se muestra en la tabla 1. Uno de los aspectos más destacados de los valores por yacimiento es que, en prácticamente ninguno de los casos, se observan diferencias estadísticamente significativas entre ambos sexos en lo que a prevalencia de exostosis se refiere (16). Un dato éste a tener en cuenta en el momento de dar una explicación histórica de la aparición y desarrollo de esta anomalía ósea.

(14) En los individuos fallecidos entre los 33 y 45 años las exostosis ocupan más de dos terceras partes del canal auditivo en un $16,66 \%$ de los casos.

(15) No obstante estos resultados no llegan a alcanzar la significación estadística en la correlación entre volumen y edad de muerte: $r$ de Pearson=0,268 $(p=0,63)$, si bien sí muestran una cierta tendencia al comportamiento descrito.

(16) Tan sólo en el yacimiento de Lomo Galeón pudieron observarse tales diferencias: $\mathrm{X}^{2}=4(\mathrm{p}<0.05)$. A pesar de ello, las variaciones en la representación numérica de individuos en cada uno de los enclaves hace que sea problemática una comparación entre ellos, por lo que el conjunto de valoraciones prefiere hacerse globalmente.

T. P., 58, n. $^{\circ} 1,2001$ 
La estimación de la prevalencia de este marcador bioantropológico atendiendo a la localización general de los yacimientos arqueológicos muestra, por el contrario, diferencias realmente significativas. Así, en el $34.35 \%$ de los individuos de los espacios costeros fue posible observar la presencia de exostosis auriculares, limitándose al $1.32 \%$ de los casos en los enclaves calificados dentro de la categoría "interior" $\left(\mathrm{X}^{2}=78.05 ; \mathrm{p}<0.0001\right)$. En el mismo sentido existe una correlación directa, estadísticamente significativa, entre la frecuencia de esta anomalía ósea y la localización espacial de los yacimientos ( $\mathrm{r}$ de Pearson=0.47; $\mathrm{p}<0.0001$ ).

En ninguno de los dos territorios definidos pudieron estimarse diferencias significativas entre hombres y mujeres (interior: $\mathrm{X}^{2}=0.34 ; \mathrm{p}=0.84 ; \mathrm{n} . \mathrm{s}$. y costa: $X^{2}=1.08 ; p=0.58$; n.s.). De este modo, en la región costera, mientras que el $37 \%$ de los individuos varones muestra esta anormalidad ósea, el porcentaje de féminas con idéntica afección se reduce ligeramente hasta alcanzar a un $30.61 \%$ del total de este grupo. En los yacimientos "interiores" de Gran canaria, de los 3 sujetos con exostosis en el canal auditivo, 2 correspondían a hombres (2/ $119 ; 1.32 \%)$ y el caso restante a una mujer (1/ $95 ; 1.05 \%)$.

\section{DISCUSIÓN}

Las exostosis auriculares constituyen un marcador óseo que tradicionalmente ha sido incluido entre los caracteres discretos localizados en el cráneo (Berry, 1975; Buikstra y Ubelaker, 1994). A pesar de ello, los estudios clínicos y experimentales han demostrado la estrecha relación entre la aparición de esta entidad y determinados factores medioambientales, lo que ha que ha quedado corroborado, además, gracias a las investigaciones bioantropológicas desarrolladas sobre distintas poblaciones arqueológicas (Capasso et alii, 1999). De este modo su consideración como carácter no métrico queda descartado en la mayor parte de los trabajos actuales, si bien, como en otros marcadores de actividad (Dutour, 1992), se continua discutiendo la existencia o no de una cierta predisposición genética para su origen y desarrollo.A tal efecto, se ha establecido con precisión que la habitual exposición del canal auditivo al agua fría provoca una reacción inflamatoria que motiva la actividad osteogénica, aunque queda aún por resolver el mecanismo por el cual tiene lugar el proceso de formación del nuevo tejido óseo. En este sentido, es un hecho bien conocido que algunas citocinas y factores de crecimiento (como las interleuquinas 1 y 6 ó los factores de necrosis tumoral $\alpha$ y $\beta$, entre otros) modulan la actividad y funcionamiento de los osteoblastos y los osteoclastos (Lerner, 1994; Mundy et alii, 1995). Así, ciertos factores de crecimiento, como los liberados durante las respuestas inflamatorias, contrarrestan la reabsorción de hueso, e inducen, de hecho, a la creación de nuevo tejido óseo en la zona afectada. Por ello es posible que el balance entre los efectos locales de crecimiento osteogénico y la reabsorción ósea durante episodios reiterados de otitis externa motivados por la exposición del canal auditivo al agua fría, desemboquen en la formación de los procesos exostósicos a los que aquí nos referimos. No obstante para la discusión que se propone desde estas páginas, y habiéndose probado la relación entre la aparición de esta anomalía ósea y determinados factores medioambientales, interesa en mayor medida conocer el alcance de este marcador para la reconstrucción de las formas de vida de las poblaciones del pasado.

Como se indicó previamente, todos los datos apuntan a que, en la aparición y progreso de las exostosis descritas, tiene un protagonismo especialmente destacado el contacto frecuente, regular y prolongado del canal auditivo con el agua fría. Por esta razón, es del todo posible asociar la notable incidencia de esta anormalidad ósea con el desarrollo por parte de estos grupos humanos de ciertas actividades que hicieran posible tal eventualidad. Resulta lógico pensar que las circunstancias descritas deben estar preferentemente vinculadas con la explotación económica del medio marino por parte de los canarios (17). Otras evidencias bioantropológicas y arqueológicas ponen de manifiesto la significación que es posible atribuir a estas labores para la consolidación del modelo productivo global, un hecho que parece venir a corroborar el estudio recogido en estas páginas. Desde este punto de vista resulta inviable seguir incluyendo estas actividades (al menos las pesqueras) como meras prácticas "complementarias" que tienen como único propósito la diversificación de alimentos que conforman la dieta alimenticia de estos grupos humanos.

(17) Podrían añadirse a tales planteamientos aquellas actividades a las que es posible atribuir un carácter "lúdico", como recogen las propias fuentes etnohistóricas: "las juelgas de la mar $i$ los baños lo tenían los más nobles por ejercicio" (Morales, 1993: 374), si bien tal relación es especialmente difícil de valorar desde un punto de vista cuantitativo. 
Las referencias aportadas por las Crónicas de la Conquista y relatos posteriores (de los siglos XVII y XVIII) en torno a las estrategias de depredación sobre el medio marino desplegadas por los canarios aportan un conjunto de evidencias que pueden ser esgrimidas para mantener las valoraciones hechas hasta el momento, así como para explicar la incidencia de este marcador bioantropológico en el repertorio óseo analizado. Por ejemplo, se recoge que: "Quando reconocían en la costa de el mar hauer cardume de pescado, se arrojaban a nado hombres i mujeres i muchachos, i la rodeaban $i$ hacían uenir serca de tierra, $i$ con esteras de juncos poniendo piedras por la parte vaxa sacaban gran cantidad de sardina i liças" (Morales, 1993: 441).

Este sistema de pesca, recurrentemente descrito por la documentación etnohistórica (18), llevaría implícito el contacto directo de parte de esta población con el medio acuático, propiciándose así la aparición y desarrollo de la anomalía ósea descrita (19). A ello habría que añadir que la temperatura media de las aguas de Canarias (Braun y Molina, 1988) (20), especialmente en determinadas épocas del año, favorecería la concurrencia de los distintos factores etiológicos que explican la frecuencia de exostosis auriculares observada.

De este modo, y siguiendo unos planteamientos adaptacionistas básicos, este marcador óseo constituiría poco más que un reflejo evidente de la interacción de este colectivo con el medio acuático.Así, los recursos del mar serían explotados siguiendo unas conductas particulares, perfectamente adecuadas a sus características específicas y a la demanda de estos productos por parte de la población prehispánica de Gran Canaria.

En cualquier caso, y dados los resultados obtenidos en esta investigación, las particularidades de este tipo de evidencias bioantropológicas, así como la complejidad de la formación social a la que se hace referencia, esta explicación resulta, cuando menos, insuficiente. A tal efecto, la heterogénea prevalencia de exostosis auriculares en los yacimientos muestreados pone de manifiesto desigualdades territoriales en esta relación entre el grupo humano y el me-

(18) Y cuyo desarrollo se encuentra probado también a partir de la representación específica de las ictiofaunas presentes en los yacimientos prehispánicos de Gran Canaria (Rodríguez, 1996).

(19) La recolección marisquera no debería haber supuesto en todos los casos el contacto frecuente y constante del canal auditivo con el agua fría, según se desprende del repertorio específico de la malacofauna localizado en los yacimientos habitaciona-les y las técnicas tradicionales de captación de estos recursos alimenticios.

(20) Oscila entre los $17-18^{\circ}$ en invierno y los $22-23^{\circ}$ en época estival.

T. P., 58, n. ${ }^{\circ} 1,2001$ dio marino. Como ha podido probarse, existen diferencias realmente significativas en los porcentajes de afección de esta anomalía ósea en función del espacio ocupado por cada uno de los enclaves analizados, de tal suerte que mientras que en las zonas próximas a la franja litoral de la Isla la presencia de exostosis en el canal auditivo concierne al $34.5 \%$ de la población asentada en estos ámbitos, entre las gentes que mantendrían su lugar de residencia en las medianías y cumbres de Gran Canaria se ve afectado un porcentaje que escasamente supera el $1 \%$.

Aceptando la directa relación entre la prevalencia de exostosis y la explotación del medio marino, tales resultados introducen un rango de territorialidad en las estrategias económicas desplegadas por esta población. Es factible estimar, a tal efecto, la existencia de notorias desemejanzas espaciales en las labores emprendidas por estos grupos humanos para la obtención de determinados recursos fundamentales. Este hecho, a su vez, puede ser interpretado como el reflejo de disimetrías territoriales en la articulación del modelo productivo global y en las estrategias seguidas para su estabilidad y perpetuación. Esta situación es especialmente llamativa en un marco geográfico de unas dimensiones relativamente reducidas, en el que no existen "barreras naturales" que impidan la movilidad de la población por los diferentes ecosistemas que ofrece este territorio insular (21).

Los trabajos previos sobre paleodieta y paleonutrición de la población prehispánica de Gran Canaria aportan, por su lado, nuevos elementos de juicio sobre los que estimar esta discusión. Los datos obtenidos a través de esta línea de investigación no se han limitado a enmarcar la economía dentro de los márgenes definidos por la tecnología y los intercambios biológicos y energéticos del grupo con la naturaleza. Atendiendo a su correlación con el resto de informaciones disponibles han hecho posible la identificación de comportamientos globales y singulares que, con relación a la dieta o la nutrición, respondan a cualquiera de las variables que como norma definen a una formación social.

Los análisis paleodietéticos llevados a cabo ponen de manifiesto como el conjunto de la población prehispánica de Gran Canaria presentaría una dieta que dependerá muy estrechamente de los productos obtenidos mediante la agricultura (González y Arnay, 1992; Velascoet alii, 1997). Este comporta-

(21) Por otro lado, las fuentes etnohistóricas aluden a la existencia de demarcaciones territoriales que compartimentan, al menos sociopoliticamente, el espacio insular. 
miento es observable en el conjunto de la población, prácticamente con independencia del lugar de procedencia del muestreo. Ello podría ser interpretado como un consumo generalizado de cereales, base fundamental de la subsistencia, con cierta independencia del área geográfica ocupada por los distintos grupos humanos. La existencia de sensibles desemejanzas en el potencial rendimiento agrícola del territorio grancanario da pie a suponer que en esta aparente homogeneidad subsistencial deben estar interviniendo, simultáneamente, otros factores. Es probable que pueda atribuirse tal responsabilidad a las redes de redistribución, convirtiéndose éstas en los cauces a través de los que se garantizan los componentes alimenticios básicos a buena parte de la población, sin que ello implique un reparto equitativo ni proporcional a las necesidades de cada sujeto.

Los análisis de elementos traza han permitido observar, además, la diversificación territorial de estrategias económicas tendentes a "equilibrar" los sistemas de producción agrícola. De este modo, los datos ofrecidos por los oligoelementos estarían reflejando una explotación intensiva del territorio, que lleva a estos grupos a mantener una economía estable a partir de un régimen agrícola plenamente consolidado, el cual será reforzado con aquellas estrategias económicas más acordes y más favorables a los intereses del grupo (Velasco, 1998). Así en el territorio litoral la posibilidad de un acceso privilegiado a las fuentes proteínicas que ofrece el medio marino favorece su explotación a las gentes que aquí se asientan. En las zonas más lejanas a la costa queda más limitada esta eventualidad, por lo que parece optarse por ampliar la gama de recursos explotados o hacer un aprovechamiento más intensivo de éstos (Velasco et alii, 1997) (22). Este "óptimo" aprovechamiento de los recursos, en el marco de un modelo agrícola desarrollado, va a colaborar en la propia estabilidad de la infraestructura económica básica y, así, en el mantenimiento de los elementos fundamentales que definen las relaciones sociales de producción observadas para estos grupos prehistóricos.

Pero quizá el aspecto más significativo en este

(22) No se puede confundir tales planteamientos con la existencia de modelos económicos diversificados en el territorio, sino tan sólo de las variables espaciales que muestra un mismo modelo de producción a fin de garantizar su estabilidad y, con ello, las relaciones de producción que lo sustentan. Por ello, la existencia de varios "modos de producción" en Gran Canaria constituye un supuesto difícilmente sostenible, más aún si se tienen presentes, en el momento de plantear tal posibilidad, los elementos que definen tal categoría. sentido, tratando de ir más allá de un planteamiento adaptacionista básico, es que la ordenación desigual de actividades económicas, con las características de las observadas para Gran Canaria, difícilmente puede darse en sociedades en las que el acceso y el control de los medios de producción no se regule basándose en parámetros de desigualdad. Este mecanismo propiciará el mantenimiento y la estabilidad de unas relaciones sociales de dependencia entre el sector social productor y el detentador del control efectivo del proceso productivo.

En la misma línea argumental, al considerar conjuntamente los datos aportados por los elementos traza y la incidencia de exostosis en los yacimientos de la isla (23) muchos de los planteamientos hechos previamente parecen quedar corroborados. De este modo, aquellos conjuntos arqueológicos que muestran una prevalencia más destacada de neoproducciones óseas en el canal auditivo son, precisamente, los que cuentan con una presencia elevada de aquellos oligoelementos indicadores de una dieta rica en productos marinos, como es el caso, por ejemplo, de los enclaves deAgaete o Gáldar (Velasco, 1998: 148-150). Por el contrario, los espacios del "interior" de la isla habían aportado unos valores de elementos traza (24) que permitían estimar un reducido consumo de este tipo de alimentos por parte de la población allí asentada. Un hecho que resultaba especialmente notorio en asentamientos como los de Guayadeque, Santa Lucía oTabacalete (Velasco, 1998: 150) y que, en este caso, coinciden con emplazamientos en los que no se evidenció ningún caso de exostosis en el canal auditivo.

Una muestra evidente de la correspondencia entre los análisis paleodietéticos y el marcador óseo que ahora centra nuestra atención puede valorarse al considerar los resultados aportados por ambas vías de análisis en el yacimiento de El Hormiguero. En este caso, las concentraciones de oligoelementos en el hueso mostraban, además de un elevado consumo de cereales, una reducida ingesta de recursos marinos por parte de este conjunto poblacional, a pesar de tener su lugar de residencia en las inmediaciones de un litoral especialmente rico para su obtención (Velasco, 1998). Estas consideraciones pueden confirmarse ahora al observar que ninguno de estos sujetos presentaba reacciones exostósicas en los canales auditivos. Sin duda será

(23) Unas valoraciones que, no obstante, siguen siendo parciales toda vez que una parte de los yacimientos aquí considerados no fueron estimados para los análisis de oligoelementos.

(24) Especialmente el coeficiente $\mathrm{Ba} / \mathrm{Sr}$. 
necesario afrontar nuevos trabajos que permitan reforzar la línea de trabajo aquí propuesta, pese a lo cual los resultados obtenidos hasta el momento resultan del todo coherentes con los planteamientos hechos en páginas previas.

La primera conclusión a la que puede llegarse, a raíz de los resultados expuestos, es que el modelo productivo de la población prehispánica de Gran Canaria muestra en su articulación territorial evidentes disimetrías, como ya habían puesto de manifiesto los análisis paleodietéticos practicados sobre este mismo conjunto poblacional. De ahí precisamente que el acceso por parte de esta población a los productos ofertados por el medio marino no pueda ser entendido de forma íntegra si no es valorado junto al resto de actividades que integran este modelo productivo, así como la participación de cada uno de ellos en su definitiva conformación. Frente a un régimen alimenticio que muestra una apariencia homogénea a escala insular, en el que los recursos agrícolas constituirían el principio fundamental de la dieta de estos grupos humanos, son los productos obtenidos a partir de las denominadas "estrategias complementarias" los que evidencian -desde un punto de vista arqueológico- un mayor índice de variabilidad y diversidad territorial.

La explicación de los hechos referidos atendiendo tan sólo a la adecuación de algunas de las estrategias económicas emprendidas por los canarios a los condicionantes impuestos por el medio insular resulta insatisfactoria, como así parecen poner de manifiesto los resultados aquí ofrecidos. Así, entre los conjuntos ubicados en las proximidades de la franja litoral de la isla existen casos en los que ninguno de los individuos examinados muestran signo alguno de reacciones exostósicas como las descritas. Es el caso, por ejemplo, de los enclaves de La Isleta o el ya mencionado de El Hormiguero, situados, además, a muy escasa distancia de la línea de costa, o el de la Cuesta de la Negra vinculado a áreas poblacionales desde las que el acceso al medio marino es sumamente sencillo. Este hecho descarta la existencia de una mera relación unívoca entre el lugar en el que se ubiquen los asentamientos y la explotación de determinados recursos alimenticios ofertados por el entorno inmediato (25).

En este sentido resulta especialmente sugerente la reflexión de P. Fournier (1992: 29) en la que

(25) La falta de verdaderas series cronológicas en todos los yacimientos estudiados impide estimar las posibles variaciones diacrónicas acaecidas en la articulación espacial del modelo productivo descrito. señala que: "los espacios de la actividad social humana deben delimitarse con base en las expresiones características de tal actividad y, en consecuencia, pueden definirse regiones culturales, político, territoriales, de recolección y de producción, por ejemplo.Aún cuando llegan a correlacionarse las regiones naturales con las de carácter socioeconómico, político o cultural, es a partir de las relaciones de explotación del entorno, es decir, de las prácticas económicas, que pueden demarcarse regiones sociales, caracterizadas por el régimen de producción". Desde este punto de vista, las formas de explotación del entorno natural de Gran Canaria, y en este caso las concernientes al medio marino, constituyen respuestas culturales, modos de trabajo, mediante las cuales esta formación social adecua el acceso a los recursos naturales a las necesidades generadas a lo largo de su desarrollo histórico (vigencia de las relaciones sociales de producción, organización sociopolítica de los territorios, mantenimiento las diferencias que rigen la gestión de los recursos naturales, etc.).

En este mismo sentido, los resultados bioantropológicos expuestos, aún a pesar de que puedan ser estimados como parciales, permiten nuevos elementos de reflexión que inciden sobre los planteamientos hechos hasta el momento.Así, ya se había señalado que para la aparición y desarrollo de las exostosis auriculares el contacto del canal auditivo con el agua fría debía ser frecuente, regular y suficientemente prolongado, hasta el punto de la inclusión de esta anomalía, por parte de algunos autores, dentro de la categoría de "enfermedades profesionales" (Dastuge y Gervais, 1992; Karegeannes, 1995; Deleyiannis et alii, 1996). Desde este punto de vista, a la desigual articulación territorial del modelo productivo en el desarrollo de al menos determinadas estrategias económicas, puede añadirse la existencia de una división social del trabajo para tal propósito.

Dado el carácter etiológico de la anomalía ósea descrita, ésta no puede ser asimilada a un acceso esporádico al medio marino, ni el reflejo de actividades ocasionales tendentes a paliar desordenes puntuales en los ciclos productivos agrícolas o ganaderos. En oposición a ello la división social del trabajo conlleva "la existencia de individuos o grupos de especialistas dedicados a la producción de determinadas clases de bienes que constituyen ramas de la producción" (Bate, 1998: 60), unas circunstancias éstas que se adecúan de mejor manera a la naturaleza de los condicionantes medioambien-

T. P., 58, n. ${ }^{\circ} 1,2001$ 
tales que, a todas luces, motivan la elevada frecuencia de exostosis auriculares en la población prehispánica de Gran Canaria. La presencia de "artesanos" o "especialistas" en esta formación social es un hecho que queda atestiguado en las fuentes etnohistóricas, con lo que una propuesta en este sentido no resulta del todo singular.

Tal eventualidad podría contribuir también a explicar el porqué de la existencia de yacimientos costeros en los que no fue posible observar la presencia de exostosis auriculares. Resulta obvio, al calor de lo señalado hasta el momento, que son las relaciones que se establecen entre los integrantes de este colectivo humano en los diferentes procesos de trabajo los que determinan qué carácter adquieren éstos en dicha sociedad. Por esta razón, los criterios que rigen esta división de tareas no responderían, al menos en principio, a condicionantes puramente técnicos o medioambientales, sino a variables de indudable carácter social.

En el mismo sentido, las circunstancias descritas permitirían dar una explicación a la desigual incidencia en la prevalencia de esta anormalidad ósea en cada uno de los enclaves funerarios sometidos a examen (26). Así, los porcentajes de individuos con exostosis auriculares variarán, según los casos, entre un 9 y un $87 \%$, mostrando una media que se sitúa en torno a un $40 \%$. Podría considerarse en este sentido, que en cada uno de estos enclaves no todos los sujetos desarrollarían similares actividades o, al menos, no todos ellos con idéntica intensidad. Quizá, dentro de cada uno de los emplazamientos estudiados la desigual ordenación territorial del modelo productivo tendría como reflejo más evidente la aludida división social del trabajo, por la que algunos sectores del grupo local llevarían a cabo determinadas tareas con un carácter más o menos especializado según los casos. Podría resultar sugerente hablar de que, al menos en el caso de la población prehispánica de Gran Canaria, las exostosis auriculares constituirían una evidencia directa en tal sentido o, en otros términos, de la presencia en determinados asentamientos de "pescadores especializados" (27).

Un hecho sobre el que sí es necesario llamar la atención en los términos propuestos, es que no pudieron observarse diferencias significativas entre ambos sexos en lo que se refiere a la presencia de

(26) Con la cautela que implica considerar yacimientos con desigual representación de individuos.

(27) Lo que, en principio, no tendría que significar la existencia de núcleos poblacionales originados para tal propósito. este marcador óseo.Así, mientras que a pesar de que el porcentaje de cráneos masculinos que mostraban esta afección es ligeramente mayor que el de los femeninos, tanto globalmente como cuando son divididos zonalmente, la prueba del chi-cuadrado aplicada a las muestras hace que tales desemejanzas no adquieran significación estadística. Por ejemplo en los yacimientos próximos a la costa, mientras que el grado de afección en los hombres supone un $37 \%$ (30/81), en las mujeres alcanza un valor de $30.6 \%(15 / 49),\left(\mathrm{X}^{2}=1,08\right.$, n.s. $)$.

Tal resultado introduce un nuevo elemento de juicio sobre el que estimar las valoraciones hechas hasta el momento. Desde este punto de vista la división social del trabajo a la que antes se hacía alusión no parece llevar implícito, al menos en este caso, una diferenciación de género en el reparto de ciertas actividades económicas. Un hecho éste especialmente llamativo toda vez que las fuentes etnohistóricas sí hacen mención expresa a la existencia de determinadas tareas que eran desempeñadas específicamente por hombres o mujeres: "Para esta sastrería y para hacer la losa que fabricaban para su servicio comun avia mugeres oficiales distrísimas" (Sosa, 1994: 284) (28).

Pero son precisamente estas mismas referencias etnohistóricas las que, en el caso concreto de la pesca, son concomitantes a la hora de apuntar la participación conjunta de ambos géneros en las labores de pesca: "Si acaso veían andar en la costa algún bando de sardinas, que hace luego señal en el agua, como eran grandes nadadores, echábanse a nado hombres, mujeres y muchachos, y cercaban el bando de las sardinas y íbanle careando para la tierra, dando palmadas o con palos en el agua. Y cuando lo tenían cerca tomaban unas esteras largas de juncos, con unas piedras atadas a la parte baja: llevándola como red, sacando a tierra mucha sardina" (Abreu, 1977: 160).

La coparticipación de hombres y mujeres en las labores pesqueras no constituye un elemento que contradiga la existencia de una especialización poblacional en esta actividad económica. A todos los efectos se trataría del reflejo evidente de una división social del trabajo por el cual determinados sectores del colectivo estarían dedicados a la producción de un tipo específico de recursos. Un reparto de tareas que, en este caso, no conllevaría una distinción de género en su desempeño, como sí

(28) Similares referencias pueden verse otras noticias etnohistóricas (Abreu, 1977: 159-160; Morales, 1993: 313, 315, 371 , 436, etc.; Torriani, 1978: 112-113). 
parece observarse en otras ocupaciones. En esta línea argumental ha de señalarse que todo apunta a que la división social del trabajo entre los canarios no respondería a un criterio único (el género, por ejemplo), sino que en su definitiva articulación serían partícipes numerosos variables, como así lo demuestra, por ejemplo, el hecho de que un sector de la población (los llamados "nobles") quedara desvinculado del ejercicio directo de la producción.

Resulta especialmente complicado dar una explicación definitiva a todos los aspectos comentados, ya que a pesar de contar con la información aportada por la documentación escrita o las evidencias bioantropológicas, son por el momento escasos los datos arqueológicos directos que pueden adicionar nuevos elementos de juicio a las consideraciones vertidas en estas páginas. No obstante, sí resulta evidente que la reconstrucción de los procesos productivos emprendidos por la población prehispánica de Gran Canaria no puede seguir estimándose como una mera respuesta adaptativa a unos supuestos condicionantes "impuestos" por el medio insular. Desde este punto de vista se puede terminar explicando la complejidad de unas normas culturales a partir de una concepción funcionalista de los procesos económicos como estrategias de adaptación a las especificidades del medio, cuya consecuencia más extrema podría ser la propuesta de la coexistencia de varios "modelos económicos" independientes en la prehistoria de esta isla. Evidentemente, los datos arqueológicos, bioantropológicos y documentales descartan tal posibilidad, manifestando, por el contrario, una formación social compleja sustentada por un modelo productivo consolidado, uno de cuyos rasgos más significativos es su diversificación territorial.

A pesar de lo controvertido que podrían llegar a ser los planteamientos hechos hasta el momento, sí ha de señalarse, a modo de conclusión, el significativo papel que puede desempeñar el estudio de los restos humanos en este tipo de análisis. Los repertorios bioantropológicos constituyen así una herramienta realmente eficaz para acceder a un conocimiento íntegro y dinámico de las poblaciones del pasado, especialmente de todos aquellos aspectos que condicionaron, de un modo u otro, su existencia.

\section{BIBLIOGRAFÍA}

Abreu Galindo, J. (1977[1632]): Historia de la Conquista de las siete Islas de Canarias. Ed. Goya. Santa Cruz de Tenerife.

T. P., 58, n. $^{\circ} 1,2001$
BATE, L.F. (1989): "Notas sobre el materialismo histórico en el proceso de investigación arqueológica". Boletín de Antropología Americana, 19: 5-29.

- (1998): El proceso de investigación en Arqueología. Crítica. Barcelona.

BERRY, A.C. (1975): "Factors affecting the incidence of non-metrical variants". Journal of Anatomy, 120: 519535.

Braun, J. y Molina, R. (1988): "El Mar". Geografía de Canarias, Tomo I. Editorial Insular Canaria, Santa Cruz de Tenerife: $17-28$.

BRothwell, D.R. (1987): Desenterrando huesos. La excavación, tratamiento y estudio de los restos del esqueleto humano. Fondo de Cultura Económica. Madrid.

- (1989): "The relationship of tooth wear and aging". En M.Y. Iscan (ed.): Age markers in the human skeleton. Charles Thomas Publisher. Illinois: 303-318.

Buikstra, J. y D.H. UbelaKer (1994): Standards for data collection from human skeletal remains. Arkansas Archeological survey research series, 44. Arkansas.

Capasso, L.; Kennedy, K. y WilczaK, C. (1999): Atlas of occupational markers on human remains. Journal of Paleontology, Monographic Publication, 3. Teramo.

Dastugue, J. (1989): "Paléopathologie et milieu". En Homme et milieu. Approches Paléoanthropologiques, Editions du CNRS. Paris: 13-16.

Dastugue, J. y Gervais, V. (1992): Paleopathologie $d u$ squelette humain. Société Nouvelle des éditions Boubée. Paris.

Deleyiannis, F.; Cockcroft, B. y Pinczower, E. (1996): "Exostoses of the external auditory canal in Oregon surfers". American Journal of Otolaryngology, 17: 303-307.

Dutour, O. (1992): "Activités physiques et squelette humain: le difficile passage de l'actuel au fossile". Bulletin et Mémories de la Société d'Anthropologie de Paris, n.s., 4, 3-4: 233-241.

Dutour, O. y Onrubia Pintado, J. (1991): "Interation homme-environnement océanique pendant la préhistoire récente des Iles Canaries: Nouvelles données paléoanthropologiques de la région de Galdar (Grande Canarie)". Centre de la Recherche, Academie des Sciences. Paris, 313: 125-130.

FourNIER, P. (1992): "Lo social y lo material en arqueología: algunos conceptos y correlatos relevantes". Boletín de Antropología Americana, 26: 25-31.

Gersztzen, P.; Gersztzen, E. y Allison, M. (1998): "Diseases of the skull inpre-Columbian South American mummies". Neurosurgery, 42: 1145-1152.

Gervais, V. (1989): "Exostoses auriculaires précolombines". En Homme et milieu. Approches paléoanthropologiques. Editions du CNRS. Paris: 107-1112.

Godelier, M. (1977): Teoría Marxista de Las Sociedades Precapitalistas. Editorial Laia. Barcelona.

- (1989): Lo Ideal y Lo Material: Pensamiento, Economía y Sociedades. Taurus. Madrid. 
GonzÁlez Antón, R. y TEJera GASPAR, A. (1990): Los aborígenes canarios. Colegio Universitario. Ediciones Istmo. Oviedo.

GonzÁlez Reimers, E. y Arnay, M. (1992): "Ancient skeletal remains of the Gran Canaria Islands: Bone histology and chemical analysis". Anthropology Anzeiger, 50: 201-215.

Hernández Gómez, C.M. y Galván Santos, B. (1997): "Materias primas y fuentes de aprovisionamiento de recursos líticos en la prehistoria de Tenerife (Islas Canarias)". II Reunió de Treball sobre aprovisionament de recursos lítics a la prehistòria (Barcelona-Gava, 1997). Rubricatum, 2: 195-203.

Hutchinson, D.; Denise, C.; Daniel, H. y Kalmus, G. (1997): "A reevaluation of the cold water etiology of external auditory exostoses". American Journal of Physical Anthropology, 103: 417-422.

ITo, M. y IKEDA, M. (1998): "Does cold water truly promote diver's ear?”. Journal Laryngology and Otology, 102 (2): 173-175.

JimÉnEz GonZÁlez, J. (1990): Los Canarios. Etnohistoria y Arqueología. Museo Arqueológico, Aula de Cultura de Tenerife. Santa Cruz de Tenerife.

KAREGEANNES, J. (1995): "Incidence of bony outgrowths of the external ear canal in U.S. Navy divers". Undersea Hyperbaric Medicine, 22: 301-306.

KENNEDY, G.E. (1986): "The relationship between auditory exostosis and cold water: A latitudinal analysis". American Journal of Physical Anthropology, 71: 401-415.

KENNEDY, K.A. (1989): "Skeletal markers of occupational stress". En M.Y. Iscan y K.A. Kennedy (eds.): Reconstruction of life from the skeleton. Alan R. Liss, Inc. New York: 129-160.

Krogman, W.M. e IscAN, M.Y. (1989): The human skeleton in forensic medicine. Charles Thomas Publisher, Illionois.

LERNER, U.H. (1994): "Regulation of bone metabolism by the kallicrein-kinin system, the coagulation cascade, and the acute-phase reactants". Oral Surgery, Oral Medicine, Oral Pathology, 78: 481-493.

MANZI, G.; SPERduti, A. y PASAREllo, P. (1991): "Behaviorinduced auditory exoxtoses in Imperial Roman society". American Journal of Physical Anthropology, 85: 253-260.

Morales Padrón, F. (1993): Canarias: Crónicas de su Conquista. Ediciones del Cabildo Insular de Gran Canaria. Las Palmas de Gran Canaria.

Mundy, G.; Boyce, B. y Hughes, D. (1995): "The effects of cytokines and growth factors on osteoblastic cells". Bone [Suppl], 17: 71-75.

Perizonius, W.R.K. (1983): "Esquema de desgaste dentario en premolares, caninos e incisivos, siguiendo el propuesto por Brothwell (1981) para molares". En C. Bouville (ed.): "Les restes humains mesolithiques de L'abri Cornille, Istres". Bulletin et Mémories de la Société d'Anthropologie de Paris, XXIII: 89-110.
Rodríguez SANTANÁ, C. (1996): La pesca entre los Canarios, Guanches y Auaritas. Ediciones del Cabildo Insular de Gran Canaria. Las Palmas de Gran Canaria.

SAKalinskas, V. y Jankauskas, R. (1993): “Clinical otosclerosis and auditory exostoses in ancient Europeans (investigation of Lithuaninan paleoosteological samples)". Journal of Laryngology and Otology, 107: 489. 491.

SosA, J. de (1994[1678]): Topografía de la Isla Afortunada de Gran Canaria. Ediciones del Cabildo Insular de Gran Canaria. Las Palmas de Gran Canaria.

Standen, V.G.; ArriazA, B. y Santoro, C. (1997): “External auditory exostosis in Prehistoric Chilean Populations: a test of the cold water hypothesis". American Journal of Physical Anthropology, 103: 119-129.

STIRLAND, A. (1992): "Diagnosis of occupationelly related paleopathology: can it be done?". En D. Otner y A. Aufderheide (eds.): Human Paleopathology. Current syntheses and future options. Smithsonian Institution Press. Washington: 40-50.

TORRIANI, L. (1978[1590]): Descripción e Historia del reino de las Islas Canarias. Goya ed. Santa Cruz de Tenerife.

Tran, L.; Grundfast, K. y Selesnick, S. (1996): "Benign lesions of the external auditory canal". Otolaryngology Clinical North American, 29 (5): 807-825.

UMeda, Y.; NAKaJima, M. y Yoshioka, H. (1989): “Surfer's ear in Japan". Laryngoscope, 99 (6): 639-641.

Velasco VázqueZ, J. (1998): "Economía y dieta de las poblaciones prehistóricas de Gran Canaria. Una aproximación bioantropológica". Complutum, 9: 137-159.

Velasco Velázquez, J.; Delgado Darias, T.; Martín RoDRíGueZ, E.; ARnay de la Rosa, M. y GonZÁlez ReIMERS, E. (2000): "Evidencias de actividades “artesanales" en la dentición de la población prehispánica de Gran Canaria". Estudios canarios. Anuario del Instituto de estudios canarios, 44: 387-405.

Velasco Vázquez, J.; González Reimers, E.; Martín RoDRÍGUeZ, E. y ARNAY DE LA Rosa, M. (1997): "Economía, dieta y territorio de las comunidades prehistóricas de Gran Canaria: los elementos traza como vehí culo de análisis". En M. Macías y J. Picazo (eds.): La enfermedad en los restos humanos arqueológicos. Servicio de publicaciones de la Universidad de Cádiz: 311 318.

Velasco Vázquez, J.; Hernández Gómez, C. y Alberto BArroso, V. (1999): "Consideraciones en torno a los sistemas productivos de las sociedades prehistóricas canarias: los modelos deTenerife y Gran Canaria".Vegueta, 4: 33-56.

Velasco VÁzquez, J. y Martín RodríGuez, E. (1998): “La Sociedad Prehistórica de Gran Canaria: Desigualdad, Apropiación y Redistribución”. Vegueta, 3: 9-28.

Whitaker, S.; Cordier, A.; Kosjakov, S. y Charbonneau, R. (1998): "Treatment of external auditory canal exostoses". Laryngoscope, 108 (2): 195-199. 